\title{
FLUORESCENCE IMAGING IN OPTICAL DIFFUSION TOMOGRAPHY
}

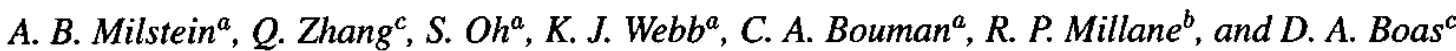 \\ ${ }^{a}$ School of Electrical and Computer Engineering, Purdue University, \\ West Lafayette, IN, 47907-1285 \\ ${ }^{b}$ Dept. of Electrical and Computer Engineering, University of Canterbury, \\ Private Bag 4800, Christchurch, New Zealand \\ ${ }^{c}$ NMR Center, Massachusetts General Hospital, Harvard Medical School, \\ 13th Street Building 149, Charlestown, MA 02129
}

\begin{abstract}
A Bayesian optimization scheme is presented for reconstructing fluorescent yield and lifetime, the absorption coefficient. and the scattering coefficient in turbid media, such as biological tissue. The proposed method utilizes measurements at both the excitation and emission wavelengths for reconstructing all unknown parameters. A tissue phantom experiment uses the inversion strategy to reconstruct a spherical heterogeneity containing indocyanine green.
\end{abstract}

\section{INTRODUCTION}

In recent years, the use of fluorescent indicators as contrast agents for in vivo imaging of tumors with near-infrared (NIR) light has shown great promise, attracting considerable new interest. In experimental studies with animal subjects $[1,2]$, fluorescence has been successfully used to visualize cancerous tissue close to the skin surface. The fluorophore, when injected into the subject, may selectively accumulate in diseased tissue due to increased blood flow from tumor neovascularization [1]. Alternatively, the agent may have different decay properties in diseased tissue, which could be useful in localizing tumors independently of fluorophore concentration [2].

In principle, modulated excitation light is launched into the biological tissue, where it is absorbed by the fluorophore. The excited fluorophore, when decaying to the ground state, emits light at a longer wavelength, and this emission is detected by a suitable measurement device. These emission data are then used to perform a tomographic reconstruction of the yield (a measure of the fluorescence efficiency) and the lifetime (the fluorescent decay constant). However, this reconstruction is complicated due to multiple scattering and absorption in human tissue [3].

A rigorous reconstruction of fluorescence property maps should also include reconstructions of absorption and scattering parameters at the excitation and emission wavelengths. This can be accomplished by use of optical diffusion tomography (ODT) [4], where boundary measurements from several sources and detectors are used to recover these unknown parameters. In addition to its usefulness in the fluorescence reconstruction, ODT may be used to localize a fluorophore as a relatively high-contrast absorber.

Several groups have addressed the fluorescence inverse problem in numerical or experimental studies. Imaging simulations with three-dimensional (3-D) [5] and two-dimensional (2-D) [6] geometries have assumed known absorption and scattering, except in some cases for the excitation wavelength absorption. Chang et al. [7] reconstructed fluorescent yield in a cylindrical tissue phantom with a heterogeneity containing Rhodamine 6G. Their study used continuous wave (CW, meaning unmodulated) light and an assumption of quasi-2-D properties in the reconstruction. Recently, Ntziachristos and Weissleder [8] used a normalized Bom approximation to reconstruct 3-D, fluorescent heterogeneities containing Cy5.5 embedded in a $2.5 \mathrm{~cm}$ diameter cylindrical tissue phantom.

Here, we present a Bayesian inversion algorithm and a measurement scheme for reconstructing all unknown fluorescent, absorption, and diffusion parameters. We use the proposed method to image a spherical heterogeneity in a tissue phantom, using transmission measurements by a $\mathrm{CW}$ imaging device. The heterogeneity contains indocyanine green (ICG), a fluorescent diagnostic agent approved by the FDA for use in the NIR range, where biomedical imaging with light is most practical. While ICG may be a strong candidate for clinical use due to its NIR excitation and emission and FDA approval, its quantum yield is relatively modest [3], which could make the emission difficult to detect in some situations. Thus, it is important to evaluate the feasibility of reconstructing ICG emission in experimental investigations.

\section{FORWARD MODEL}

The transport of modulated light (at $\omega$, with $e^{j \omega t}$ variation) in a fluorescent, diffusely scattering medium can be modeled using coupled diffusion equations [5]:

$$
\begin{array}{r}
\nabla \cdot\left[D_{x}(r) \nabla \phi_{x}(r, \omega)\right]-\left[\mu_{a_{x}}(r)+j \omega / c\right] \phi_{x}(r, \omega) \\
=-S_{x}(r, \omega) \\
\nabla \cdot\left[D_{m}(r) \nabla \phi_{m}(r, \omega)\right]-\left[\mu_{a_{m}}(r)+j \omega / c\right] \phi_{m}(r, \omega) \\
=-\phi_{x}(r, \omega) \gamma(r, \omega)[1-j \omega \tau(r)] \\
\gamma(r, \omega)=\eta \mu_{a_{x \rightarrow m}}(r) \frac{1}{1+[\omega \tau(r)]^{2}}
\end{array}
$$


where the subscripts $x$ and $m$ respectively denote excitation and emission wavelengths, $\phi(r, \omega)$ is the photon fluence, and $S_{x}(r, \omega)$ is the excitation source distribution. The optical parameters are the diffusion coefficient $D(r)$ and the absorption coefficient $\mu_{a}(r)$. The fluorescence parameters are the lifetime $\tau(r)$ and the fluorescent yield $\eta \mu_{a_{x \rightarrow m}}(r)$. Note the right hand side of (2), where the light absorbed by fluorophores and subsequently emitted at the emission wavelength is incorporated into an effective source term.

\section{INVERSION}

In the most general case, the unknown parameters in (1) and (2) are $\mu_{a_{x}}, \mu_{a_{m}}, D_{x}, D_{m}, \tau$, and $\eta \mu_{a_{x \rightarrow m}}$. Reconstructions of the $D_{x}$ and $\mu_{a_{e}}$ images may be obtained using data from sources and detection at the excitation wavelength. Similarly, $D_{m}$ and $\mu_{a_{m}}$ may be obtained using data from sources and detection at the emission wavelength. Finally, sources at the excitation wavelength and detectors filtered at the emission wavelength can record fluorescence. The $D$ and $\mu_{a}$ reconstructions may then be utilized in the fluorescence inversion.

We formulate each image reconstruction as a Bayesian inverse problem and compute the maximum $a$ posteriori estimate, given by

$$
\hat{\mathbf{x}}=\arg \max _{\mathbf{x} \geq 0}\{\log p(\mathbf{y} \mid \mathbf{x})+\log p(\mathbf{x})\}
$$

where $\mathbf{x}$ represents a general unknown image, $\mathbf{y}$ is the measurement vector, $p(\mathbf{y} \mid \mathbf{x})$ is the data likelihood, and $p(\mathbf{x})$ is the prior density for the image. The data likelihood can be formed from a Gaussian model by considering the physical properties of the measurement system [9]. For the prior density $p(\mathbf{x})$, we use the generalized Gaussian Markov random field (GGMRF) model, which has been found to enforce smoothness in the solution while preserving sharp edge transitions [9]. It is given by

$$
p(\mathbf{x})=\frac{1}{\sigma^{N} z(p)} \exp \left[-\frac{1}{p \sigma^{p}} \sum_{\{i, j\} \in \mathcal{N}} b_{i-j}\left|x_{i}-x_{j}\right|^{p}\right],
$$

where $x_{i}$ denotes the image node values, $N$ is the dimension of the image vector, $\sigma$ is a normalization parameter, and $p$ controls the sharpness of the edge transition. The set $\mathcal{N}$ consists of all pairs of neighboring grid points, and the neighborhood weighting coefficients $b_{i-j}$ are assigned to be inversely proportional to the node separation.

Assume for simplicity that $D_{x}$ and $D_{m}$ are known (a restriction easily removed by extending the concepts below to include scattering parameters). Reconstruction of the unknowns can be achieved by forming and optimizing three cost functions:

$$
\begin{aligned}
& \hat{\mu_{a_{x}}}=\arg \min _{\mu_{\mathbf{s}_{x}} \geq 0, \alpha_{x, x}}\left\{\frac{1}{\alpha_{x, x}}\left\|y_{x, x}-f_{x, x}\left(\mu_{a_{x}}\right)\right\|_{\Lambda_{x, x}}^{2}\right.
\end{aligned}
$$

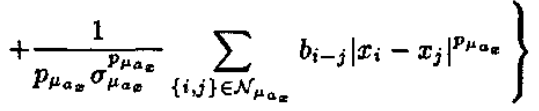

$$
\begin{aligned}
& \hat{\mu_{\mathbf{a}_{m}}}=\arg \min _{\mu_{\mathbf{a}_{m}} \geq 0, \alpha_{m, m}}\left\{\frac{1}{\alpha_{m, m}}\left\|\mathbf{y}_{m, m}-\mathbf{f}_{m, m}\left(\mu_{\alpha_{m}}\right)\right\|_{\Lambda_{m, m}}^{2}\right. \\
& \left.+\frac{1}{p_{\mu_{a_{m}}} \sigma_{\mu_{a_{m}}}^{p_{\mu_{a_{m}}}}} \sum_{\{i, j\} \in \mathcal{N}_{\mu_{a_{m}}}} b_{i-j}\left|x_{i}-x_{j}\right|^{p_{\mu_{a_{m}}}}\right\}
\end{aligned}
$$

\section{(7)}

$$
\begin{aligned}
{[\hat{\gamma}, \hat{\tau}]=\arg \min _{\gamma, \tau \geq \mathbf{0}, \alpha_{\varepsilon, m}} } & \left\{\frac{1}{\alpha_{x, m}}\left\|\mathbf{y}_{x, m}-\mathbf{f}_{x, m}(\gamma, \tau)\right\|_{\Lambda^{\prime}, m}^{2}\right. \\
& +\frac{1}{p_{\gamma} \sigma_{\gamma}^{p_{\gamma}}} \sum_{\{i, j\} \in \mathcal{N}_{\gamma}} b_{i-j}\left|x_{i}-x_{j}\right|^{p_{\gamma}} \\
& \left.+\frac{1}{p_{\tau} \sigma_{\tau}^{p_{\tau}}} \sum_{\{i, j\} \in \mathcal{N}_{\tau}} b_{i-j}\left|x_{i}-x_{j}\right|^{p_{\tau}}\right\}
\end{aligned}
$$

where the subscripts $(x, x),(m, m)$, and $(x, m)$ specify the use of excitation or emission wavelength in the sources (the first in$\mathrm{dex}$ ) and the detectors (the second index), $\mathrm{f}$ is the appropriate forward operator, $\Lambda$ is the inverse of a diagonal covariance matrix, and $\alpha$ is an estimated parameter which scales the noise variance $[9,4]$. The optimization can be performed using the iterative coordinate descent (ICD) algorithm $[9,4]$, a sequential single-site update scheme similar to the Gauss-Seidel method used in other problems. Note that the parameterization of the fluorescent properties as $\gamma$ and $\tau$ is useful because, in a sequential optimization scheme, it takes advantage of the inherent linearity of the fluorescence inverse problem while allowing regularization to be applied to $\tau$ directly.

\section{EXPERIMENT}

To verify the proposed reconstruction method, we performed fuorescence measurements. We recorded the data using a $\mathrm{CW}$ imaging device and a versatile phantom box (shown in Figure 1), both described in detail elsewhere [10]. The $\mathrm{CW}$ instrument has sources availabie at both $690 \mathrm{~nm}$ and $830 \mathrm{~nm}$. While $690 \mathrm{~nm}$ excitation is not ideal for ICG, a published excitation spectrum [11] indicates that the detected $830 \mathrm{~nm}$ emission intensity, using $690 \mathrm{~nm}$ excitation, is about $30 \%$ of the maximum value (obtained using 780 $\mathrm{nm}$ excitation). The box was adjusted to internal dimensions of $16 \times 16 \times 3.9 \mathrm{~cm}$, where the last dimension is vertical thickness. As shown in Figure 2, 9 source fibers were connected to the bottom plate, and 14 detector fibers were connected to the top plate. A hollow, surface frosted glass sphere of diameter $2 \mathrm{~cm}$ was mounted just beneath the top plate. This sphere was connected to a closed circulation channel via thin rubber tubes leading outside the box, allowing fluorophore solution to be titrated into the sphere from an external reservoir over the course of the experiment.

The box was filled with a solution of $0.4 \%$ Intralipid. Assuming $690 \mathrm{~nm}$ excitation and $830 \mathrm{~nm}$ emission, this results in background values of approximately $D_{x}=0.071 \mathrm{~cm}, D_{m}=0.082$ $\mathrm{cm}, \mu_{a_{s}}=0.006 \mathrm{~cm}^{-1}$, and $\mu_{a_{m}}=0.03 \mathrm{~cm}^{-1}[12,13]$. The sphere was initially filled with the same solution. Measurements at $690 \mathrm{~nm}$ and $830 \mathrm{~nm}$ were made on this essentially homogeneous slab for calibration purposes. ICG was titrated into the sphere at a concentration of $1.0 \mu \mathrm{mol} / \mathrm{L}$. For the purposes of reconstructing $\mu_{a_{x}}$, measurements using $690 \mathrm{~nm}$ sources (with no optical bandpass filters installed in the detectors) were recorded. Similarly, 830 $\mathrm{nm}$ data were recorded (with no filters) for reconstructing $\mu_{a_{m}}$. Finally, fluorescence measurements were made using $690 \mathrm{~nm}$ source excitation and $830 \mathrm{~nm}$ bandpass filters with $12 \mathrm{~nm}$ FWHM (Newport 10LF10-830) installed in the detectors to detect the emission. In addition, baseline measurements, with $690 \mathrm{~nm}$ sources and filters present, were made on the homogeneous phantom for the purpose of finding and subtracting certain compting effects such as background light or leakage of the $690 \mathrm{~nm}$ excitation through the filter. Due to a limited number of filters, only 9 of the 14 detectors 
were used for recording the filtered fluorescence measurements (as shown in Figure 2).

Before applying the reconstruction algorithm, a simple experimental calibration procedure was performed. Synthetic data for a homogeneous phantom with $D_{x}=0.071 \mathrm{~cm}, D_{m}=0.082 \mathrm{~cm}$ $\mu_{a_{\mathrm{v}}}=0.006 \mathrm{~cm}^{-1}$, and $\mu_{a_{m}}=0.03 \mathrm{~cm}^{-1}$ were computed on a $33 \times 33 \times 17$ grid using multigrid finite differences. These computed data were then divided by the corresponding $690 \mathrm{~nm}$ and $830 \mathrm{~nm}$ experimental data measured on the homogeneous phantom. The resulting calibration factors were then multiplied by the raw absorption measurements recorded with the ICG present. This calibration procedure reduces problems caused by the unknown scaling of the measurements or possibly nonuniform source and detector coupling to the medium. However, no calibration was performed for the fluorescence measurements, as relevant calibration data (with filters present) were not available.

The reconstructions of $\mu_{a_{x}}$ and $\mu_{a_{m}}$ are shown in Figures 3 and 4 , respectively. For each inversion, a volume representing the whole box was discretized into $33 \times 33 \times 17$ voxels. The ICD algorithm, using $p=1.2$ and $\sigma=0.01$, was run for 20 iterations on a $927 \mathrm{MHz}$ Pentium III workstation, taking approximately 15 minutes. The resulting $\mu_{a_{z}}$ image shows a heterogeneity with accurate shape, though with artifacts present in the region close to the top plate. The $\mu_{a_{m}}$ image shows very little contrast, consistent with our expectation that ICG absorption should be higher at the excitation wavelength.

Figure 5 shows the reconstructed fluorescent yield $\eta \mu_{a_{x \rightarrow m}}$. Making use of the reconstructed $\mu_{a_{z}}$ and $\mu_{a_{m}}$, the ICD algorithm, using $p=1.2$ and $\sigma=1.0$, was run for 30 iterations (about 4 minutes). The iterations were computationally inexpensive due to the linearity of the fluorescence inverse problem. As a result of using uncalibrated data, the image is in arbitrary units. (The large value of $\sigma$ also reflects the arbitrary scale of the image.) Though no quantitative interpretation is possible, the reconstructed fluorophore has accurate shape and location.

\section{CONCLUSION}

We have presented a Bayesian inversion strategy for reconstructing fluorescence, absorption, and scattering properties, and we have used the method in a tissue phantom experiment to image fluorescence from ICG in a spherical heterogeneity. The results show potential for use of fluorescence, absorption, or possibly both as a tool for localizing ICG in clinical diagnostic applications.

\section{ACKNOWLEDGMENTS}

This work was funded by the National Science Foundation under contract CCR-0073357. In addition, we thank Tina Chaves for her assistance with the laboratory equipment.

\section{REFERENCES}

[1] J. S. Reynolds, T. L. Troy, R. H. Mayer, A. B. Thompson, D. J. Waters, K. K. Cornell, P. W. Snyder, and E. M. SevickMuraca, "Imaging of spontaneous canine mammary tumors using fluorescent contrast agents," Photochem. Photobiol., vol. 70, pp. 87-94, 1999.

[2] R. Cubeddu, G. Canti, A. Pifferi, P. Taroni, and G. Valentini, "Fluorescence lifetime imaging of experimental tumors in hematoporphyrin derivative-sensitized mice," Photochem. Photobiol., vol. 66, pp. 229-236, 1997.

[3] E. M. Sevick-Muraca, G. Lopez, J. S. Reynolds, T. L. Troy, and C. L. Hutchinson, "Fluorescence and absorption contrast mechanisms for biomedical optical imaging using frequency-domain techniques," Photochem. Photobiol., vol. 66, no. 1, pp. 55-64, 1997.

[4] A. B. Milstein, S. Oh, J. S. Reynolds, K. J. Webb, C. A. Bouman, and R. P. Millane, "Three-dimensional Bayesian optical diffusion tomography with experimental data," Opt. Lett., vol. 27, no. 2, pp. 95-97, January 152002.

[5] R. Roy and E. M. Sevick-Muraca, "Three-dimensional unconstrained and constrained image-reconstruction techniques applied to fiuorescence, frequency-domain photon migration," Appl. Opt., vol. 40, no. 13, pp. 2206-2215, May 2001.

[6] M. A. O'Leary, D. A. Boas, X. D. Li, B. Chance, and A. G. Yodh, "Fluorescence lifetime imaging in turbid media," Opt. Lett., vol. 21, no. 2, pp. 158-160, January 1996.

[7] J. Chang, H. L. Graber, and R. L. Barbour, "Luminescence optical tomography of dense scattering media," J. Opt. Soc. Am. A, vol. 14, pp. 288-299, January 1997.

[8] V. Ntziachristos and R. Weissleder, "Experimental threedimensional fluorescence reconstruction of diffuse media by use of a normalized Born approximation," Opt. Lett., vol. 26, no. 12, pp. 893-895, June 2001.

[9] J. C. Ye, K. J. Webb, C. A. Bouman, and R. P. Millane, "Optical diffusion tomography using iterative coordinate descent optimization in a Bayesian framework," J. Opt. Soc. Am. A, vol. 16, no. 10, pp. 2400-2412, October 1999.

[10] Q. Zhang, T. J. Brukilacchio, T. Gaudett, L. Wang, A. Li, and D. A. Boas, "Experimental comparison of using continuouswave and frequency-domain diffuse optical imaging systems to detect heterogeneities," in Proc. SPIE, June 2001, vol. 4250, pp. 219-238.

[11] R. H. Mayer, J. S. Reynolds, and E. M. Sevick-Muraca, "Measurement of the fluorescence lifetime in scattering media by frequency-domain photon migration," Appl. Opt., vol. 38, pp. 4930-4938, August 1999.

[12] H. J. van Staveren, C. J. M. Moes, J. van Marie, S. A. Prahl, and M. J. C. van Gemert, "Light scattering in Intralipid-10\% in the wavelength range of $400-1100 \mathrm{~nm}$," Appl. Opt., vol. 30, no. 31, pp. 4507-4514, November 1991.

[13] George M. Hale and Marvin R. Querry, "Optical constants of water in the 200-nm to 200- $\mu$ m wavelength region," Appl. Opt., vol. 12, no. 3, pp. 555-563, March 1973. 


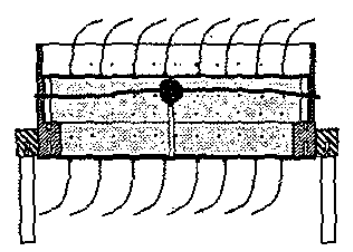

Fig. 1. Phantom box schematic, showing fibers and spherical heterogeneity.

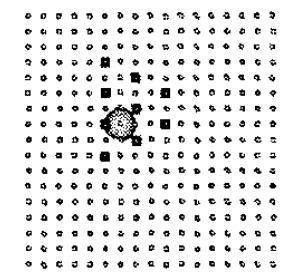

(a) Bottom plate (sources)

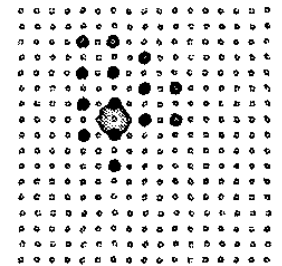

(b) Top plate (detectors)
Fig. 2. Source and detector layout. The blackened detector symbols represent detector positions used in the fluorescence measurements. The relative location of the sphere is also depicted.

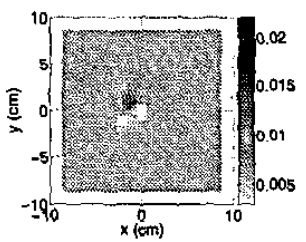

(a) $z=-1.04 \mathrm{~cm}$

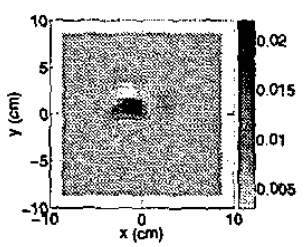

(c) $z=0.53 \mathrm{~cm}$

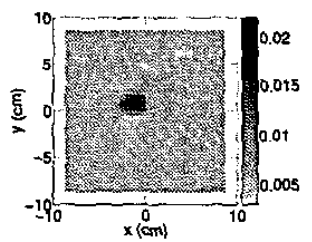

(b) $z=-0.26 \mathrm{~cm}$

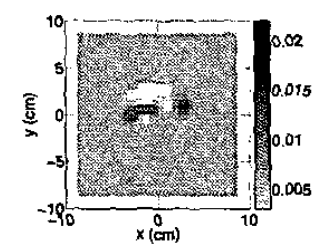

(d) $z=1.31 \mathrm{~cm}$

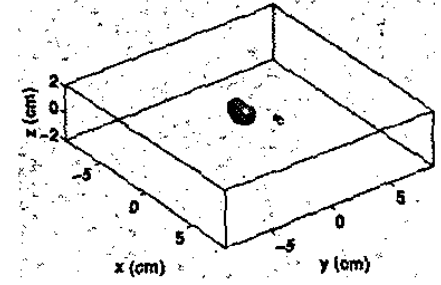

(e) $\mu_{\alpha_{x}}=0.016 \mathrm{~cm}^{-1}$ isosurface

Fig. 3. Reconstructions of $\mu_{a_{\sigma}}$ in $\mathrm{cm}^{-1}$

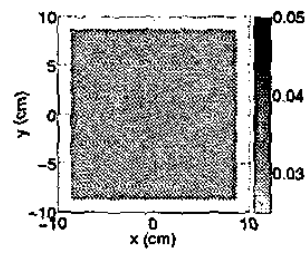

(a) $z=-1.04 \mathrm{~cm}$

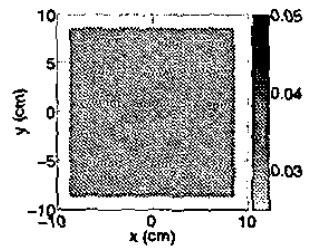

(c) $z=0.53 \mathrm{~cm}$

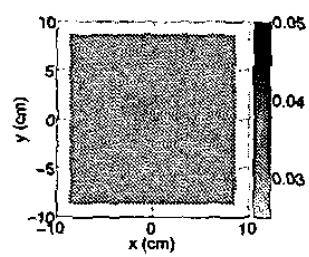

(b) $z=-0.26 \mathrm{~cm}$

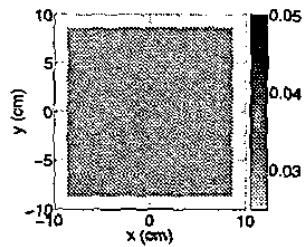

(d) $z=1.31 \mathrm{~cm}$
Fig. 4. Reconstructions of $\mu_{a_{m}}$ in $\mathrm{cm}^{-1}$

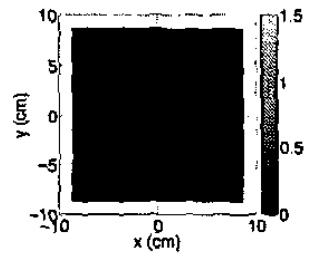

(a) $z=-1.04 \mathrm{~cm}$

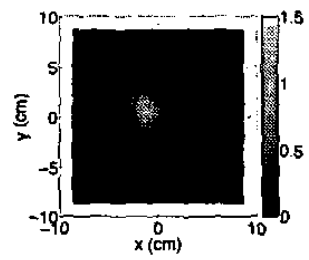

(c) $z=0.53 \mathrm{~cm}$

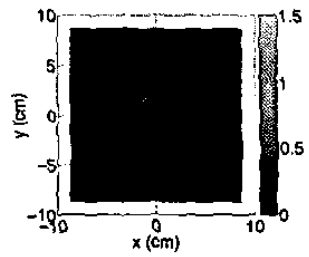

(b) $z=-0.26 \mathrm{~cm}$

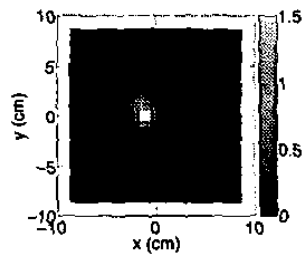

(d) $z=1.31 \mathrm{~cm}$

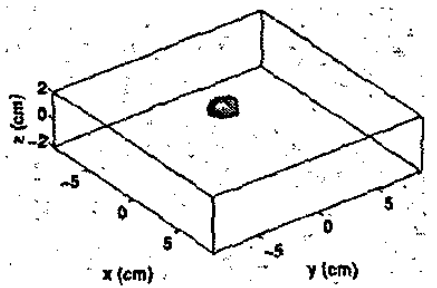

(e) Arbitrarily scaled $\eta \mu_{a_{e-m}}=0.75$ isosurface

Fig. 5. Reconstructions of $\eta \mu_{a_{e-m}}$ in arbitrary units 